\title{
A new iterative method based solution for fractional Black-Scholes option pricing equations (BSOPE)
}

\author{
Rajarama Mohan Jena $^{1} \cdot$ S. Chakraverty ${ }^{1}$
}

C Springer Nature Switzerland AG 2018

\begin{abstract}
In this manuscript, a new expansion technique namely residual power series method is used for finding the analytical solution of the Fractional Black-Scholes equation with an initial condition for European option pricing problem. The Black-Scholes formula is important for estimating European call and put option on a non-dividend paying stock in particular when it contains time-fractional derivatives. The fractional derivative is defined in Caputo sense. This technique is based on fractional power series expansion. The convergence analysis of the present method is also deliberated. Example problems are given to examine the efficacy of the proposed method. Obtained solutions are compared with exact solutions solved by other techniques which demonstrate that the present method is robust and easy to implement for other fractional problems arising in science and engineering.
\end{abstract}

Keywords Black-Scholes option pricing equation · Residual power series · Fractional derivative

Mathematics Subject Classification 35R11 · 91G80 · 91B25

\section{Introduction}

Pricing of options is a critical element of mathematical finance problems. The important thought of their examinations is that one did not have to evaluate the regular return of a stock with a specific end goal to value an option written on that stock. In 1973, the familiar theoretical widespread valuation formula for options was derived with the aid of Fischer Black and Myron Scholes [1] which received them the 1997 Nobel Prize in Economics. The FBSE which is a second-order parabolic equation deals with the estimation of financial derivatives. Such equations support the usage of the no-arbitrage principle also. Hence, the BS equation is utilized for estimating call and put options on a paying stock [2]. In this association, various researchers have performed innovative work which includes Hilfer [3], Podlubny [4], Caputo [5], Miller and Ross [6], Kilbas et al. [7], Heydari et al. [8, 9] and others.
As such in this article, fractional BSOPE is considered as

$\frac{\partial^{\alpha} w}{\partial t^{\alpha}}+\frac{\sigma^{2} x^{2}}{2} \frac{\partial^{2} w}{\partial x^{2}}+s(t) x \frac{\partial w}{\partial x}-s(t) w=0, \quad(x, t) \in R^{+} \times(0, T)$,

where $w(x, t), s(t), \sigma(x, t), T$ and $t$ denote the European call option price, interest rate, volatility function, maturity and time respectively. The payoff functions are

$w_{\text {call }}(x, t)=\max (0, x-E), \quad w_{\text {put }}(x, t)=\max (0, E-x)$,

where $w_{\text {call }}(x, t)$ and $w_{\text {put }}(x, t)$ signify the values of European call and put option respectively. $E$ represents the expiration price of the option. The fractional BSOPE has been examined with the help of various techniques such as Laplace Transform Method (LTM) [10], Homotopy Perturbation Method (HPM) [11], Homotopy Analysis Method (HAM) [11], Sumudu Transform Method (STM) [12], Projected Differential Transformation Method (PDTM) [13],

S.Chakraverty, sne_chak@yahoo.com; Rajarama Mohan Jena, rajarama1994@gmail.com | 'Department of Mathematics, National Institute of Technology Rourkela, Rourkela 769008, India. 
Adomian Decomposition Method (ADM) with conformal derivative and Modified Homotopy Perturbation Method (MHPM) [14], Multivariate Padé Approximation [15] and ADM [16]. Recently fractional order European Vanilla option pricing model has also been studied by Yavuz and Özdemir [17]. These methods have their particular limits and inadequacies. Also, these methods require tremendous computational work and take high running time. As regards, RPSM is found to be an efficient and effective method initially recommended by the mathematician Abu Arqub [18]. The RPSM has been implemented in the generalized Lane-Emden equation [19], fractional KdV-Burgers equation [20] and fractional foam drainage equation [21]. Moreover, RPSM has also been effectively applied to the time-space-fractional Benny-Lin equation [22].

In this article, RPSM has been implemented for solving the fractional BS European option pricing equation. The performance and precision of the present method are studied by comparing the solution of the titled problem solved by RPSM and other analytical methods. However, to the best of the authors' information, the time-fractional BS equation has not yet been solved by RPSM.

\section{Preliminaries of fractional calculus and RPSM}

Definition 2.1 $[4,6]$ The Abel-Riemann (A-R) fractional derivative operator $D^{\alpha}$ of order $\alpha$ is defined as

$D^{\alpha} u(x)=\left\{\begin{array}{lc}\frac{d^{m}}{d x^{m}} u(x), & \alpha=m, \\ \frac{1}{\Gamma(m-\alpha)} \frac{d}{d x^{m}} \int_{0}^{x} \frac{u(t)}{(x-t)^{\alpha-m+1}} d t, & m-1<\alpha<m .\end{array}\right.$

where $m \in Z^{+}, \alpha \in R^{+}$.

Definition 2.2 $[4,6]$ The integral operator $J^{\alpha}$ in A-R sense is defined as

$J^{\alpha} u(x)=\frac{1}{\Gamma(\alpha)} \int_{0}^{x}(x-t)^{\alpha-1} u(t) d t, \quad t>0, \quad \alpha>0$.

Following Podlubny [4] we may have

$$
\begin{aligned}
& J^{\alpha} t^{m}=\frac{\Gamma(m+1)}{\Gamma(m+\alpha+1)} t^{m+\alpha} . \\
& D^{\alpha} t^{m}=\frac{\Gamma(m+1)}{\Gamma(m-\alpha+1)} t^{m-\alpha} .
\end{aligned}
$$

Definition $2.3[4,5]$ The Caputo fractional derivative operator $D^{\alpha}$ is well-defined as

${ }^{C} D^{\alpha} u(x)=\left\{\begin{array}{lc}\frac{1}{\Gamma(m-\alpha)} \int_{0}^{x} \frac{u^{m}(t)}{(x-t)^{\alpha-m+1}} d t, & m-1<\alpha<m, \\ \frac{d^{m}}{d t^{m}} u(x), & \alpha=m .\end{array}\right.$

Definition 2.4 [4-6]

(a) $D_{t}^{\alpha} J_{t}^{\alpha} g(t)=g(t)$,

(b) $J_{t}^{\alpha} D_{t}^{\alpha} g(t)=g(t)-\sum_{k=0}^{m} g^{(k)}\left(0^{+}\right) \frac{t^{k}}{k !}$

$$
\text { for } m-1<\alpha \leq m \text {, and } t>0 \text {. }
$$

Definition 2.5 A series of the form

$$
\begin{aligned}
& \sum_{k=0}^{\infty} a_{k}\left(t-t_{0}\right)^{k \alpha}=a_{0}+a_{1}\left(t-t_{0}\right)^{\alpha}+a_{2}\left(t-t_{0}\right)^{2 \alpha}+\cdots \\
& \quad \text { for } 0 \leq n-1<\alpha \leq n, t \geq t_{0}
\end{aligned}
$$

is called FPSE at $t=t_{0}$, where $a_{k}$ is the coefficient of series.

Theorem 2.1 If $f(t)=\sum_{k=0}^{\infty} a_{k}\left(t-t_{0}\right)^{k \alpha}$ and $D^{k \alpha} f(t) \in C$ $\left(t_{0}, t_{0}+R\right)$ for $k=0,1,2, \ldots$ then the value of $a_{k}$ in Eq. (10) is given by $a_{k}=\frac{D^{k \alpha} f\left(t_{0}\right)}{\Gamma(k \alpha+1)}$.

Definition 2.6 An FPSE about $t=t_{0}$ of the form $\sum_{k=0}^{\infty} b_{k}(x)\left(t-t_{0}\right)^{k \alpha}$ is called multiple FPSE about $t=t_{0}$, where $b_{k}$ 's are coefficients of the series.

\section{RPS solution for FBSE}

Let us consider the FBSE as [23]

$D_{t}^{\alpha} w=D_{x}^{2} w+(k-1) D_{x} w-k w, \quad 0<\alpha \leq 1$,

with IC

$w(x, 0)=\max \left(e^{x}-1,0\right)$.

\subsection{Procedure of RPS solution}

Step 1 Let us assume that FPSE of Eq. (11) with IC Eq. (12) about the point $t=t_{0}$ is written as

$w(x, t)=\sum_{k=0}^{\infty} b_{k}(x) \frac{t^{\alpha k}}{\Gamma(\alpha k+1)}, \quad 0<\alpha \leq 1, \quad 0 \leq t$. 
In order to evaluate the value of $w(x, t)$, let $w_{m}(x, t)$ signifies the mth truncated series of $w(x, t)$ as

$w_{m}(x, t)=\sum_{k=0}^{m} b_{k}(x) \frac{t^{\alpha k}}{\Gamma(\alpha k+1)}, \quad 0<\alpha \leq 1, \quad 0 \leq t$.

For $m=0$, the 0th RPS solution of $w(x, t)$ may be written as

$w_{0}(x, t)=b_{0}(x)=\max \left(0, e^{x}-1\right)$.

Using Eqs. (15) and (14) can be modified as

$$
\begin{gathered}
w_{m}(x, t)=b_{0}(x)+\sum_{k=1}^{m} b_{k}(x) \frac{t^{\alpha k}}{\Gamma(\alpha k+1)}, \quad 0<\alpha \leq 1, \\
0 \leq t, \quad m=1,2,3, \ldots
\end{gathered}
$$

So mth RPS solution can be evaluated after obtaining all $b_{k}(x), k=1,2, \ldots, m$.

Step 2 Let us consider the residual function (RF) of Eq. (11) as

$\operatorname{res}(x, t)=D_{t}^{\alpha} w-D_{x}^{2} w-(k-1) D_{x} w+k w$,

and mth RF may be written as

$$
\begin{aligned}
& \operatorname{res}_{m}(x, t)=\frac{\partial^{\alpha} w_{m}(x, t)}{\partial t^{\alpha}}-\frac{\partial^{2} w_{m}(x, t)}{\partial x^{2}} \\
& -(k-1) \frac{\partial w_{m}(x, t)}{\partial x}+k w_{m}(x, t), \quad m=1,2,3, \ldots
\end{aligned}
$$

Some useful results about res $m(x, t)$ have been included in $[21,22]$ which are given below

$$
\begin{aligned}
& \operatorname{res}^{\text {i. }}(x, t)=0 . \\
& \text { ii. } \\
& \operatorname{Lim}_{m \rightarrow \infty} \operatorname{res}_{m}(x, t)=\operatorname{res}(x, t) . \\
& D_{t}^{i \alpha} \text { iii. } r e s(x, 0)=D_{t}^{i \alpha} \operatorname{res}_{m}(x, 0)=0, \quad i=0,1,2, \ldots, m .
\end{aligned}
$$

Step 3 Putting Eq. (16) into Eq. (18) and calculating $D_{t}^{(k-1) \alpha} \operatorname{res}_{m}(x, t), \quad k=1,2, \ldots$ at $t=0$, together with the above three results, we have the following algebraic systems

$D_{t}^{(k-1) \alpha} \operatorname{res}_{m}(x, 0), \quad 0<\alpha \leq 1, \quad k=1,2, \ldots$

Step 4 By solving Eq. (20), we can get the coefficients $b_{k}(x), \quad k=1,2, \ldots, m$. Thus mth RPS approximate solution is derived.

\section{Convergence analysis}

Lemma 1 [4] If $f(x)$ is a continuous function and $\alpha, \beta>0$ then $\left.\right|_{c} ^{\alpha} l_{c}^{\beta} f(x)=l_{c}^{\alpha+\beta} f(x)=\left.\left.\right|_{c} ^{\beta}\right|_{c} ^{\alpha} f(x)$.

\section{Theorem 4.1}

(a) If the FPS of the form $\sum_{n=0}^{\infty} a_{n} x^{n \alpha}, \quad x \geq 0$ converges at $x=x_{\gamma}$ then it converges absolutely $\forall x$ satisfying $|x|<\left|x_{1}\right|$.

(b) If the FPS diverges at $x=x_{\gamma}$ then it will diverge $\forall x$ such that $|x|>\left|x_{1}\right|$.

Theorem 4.2 [22] For $0 \leq n-1<\alpha \leq n$, suppose $D_{t}^{r+k \alpha}, D_{t}^{r+(k+1) \alpha} \in C\left[R, t_{0}\right] \times\left[R, t_{0}+R\right]$,

$$
\begin{aligned}
& \left(l_{t}^{r+k \alpha} D_{t}^{r+k \alpha} u\right)(x, t)-\left(l_{t}^{r+(k+1) \alpha} D_{t}^{r+(k+1) \alpha} u\right)(x, t) \\
& =\frac{\left(t-t_{0}\right)^{r+k \alpha}}{\Gamma(r+k \alpha+1)} D_{t}^{r+k \alpha} u\left(x, t_{0}\right),
\end{aligned}
$$

where $D_{t}^{r+k \alpha}=\underset{\substack{\left.D_{t} D_{t} D_{t} \ldots\right) \\ r \text { times }}}{\left(D_{t}^{\alpha} D_{t}^{\alpha} D_{t}^{\alpha} \ldots\right) \text {.times }}$

Proof Using Lemma 1,

$$
\begin{aligned}
& \left(l_{t}^{r+k \alpha} D_{t}^{r+k \alpha} u\right)(x, t)-\left(l_{t}^{r+(k+1) \alpha} D_{t}^{r+(k+1) \alpha} u\right)(x, t) \\
& \quad=l_{t}^{r+k \alpha}\left(\left(D_{t}^{r+k \alpha} u\right)(x, t)-\left(l_{t}^{\alpha} D_{t}^{r+(k+1) \alpha} u\right)(x, t)\right) \\
& \quad=I_{t}^{r+k \alpha}\left(\left(D_{t}^{r+k \alpha} u\right)(x, t)-\left(l_{t}^{\alpha} D_{t}^{\alpha}\right)\left(D_{t}^{r+k \alpha} u\right)(x, t)\right) \\
& \quad=l_{t}^{r+k \alpha}\left(\left(D_{t}^{r+k \alpha} u\right)\left(x, t_{0}\right)\right)=\frac{\left(t-t_{0}\right)^{r+k \alpha}}{\Gamma(r+k \alpha+1)} D_{t}^{r+k \alpha} u\left(x, t_{0}\right) .
\end{aligned}
$$

Theorem 4.3 [22] Let $w(x, t), D_{t}^{k \alpha} w(x, t) \in C\left[R, t_{0}\right] \times$ $\left[R, t_{0}+R\right]$ where $k=0,1,2, \ldots, N+1$ and $j=0,1,2$, $\ldots, n-1$. Also $D_{t}^{k \alpha} w(x, t)$ may be differentiated $n-1$ times concerning " $t$ ". Then

$w(x, t) \cong \sum_{j=0}^{n-1} \sum_{i=0}^{N} W_{j+i \alpha}(x)\left(t-t_{0}\right)^{j+i \alpha}$,

where $W_{j+i \alpha}(x)=\frac{D_{t}^{j+i \alpha} w\left(x, t_{0}\right)}{\Gamma(j+i \alpha+1)}$. Also, $\exists$ a value $\varepsilon, 0 \leq \varepsilon \leq t$, the error term has the term as follows,

$\left\|E_{N}(x, t)\right\|=\sup _{t \in[0, T]}\left|\sum_{j=0}^{n-1}\left[\frac{D^{j+(N+1) \alpha} w(x, \varepsilon)}{\Gamma((N+1) \alpha+j+1)} t^{(N+1) \alpha+j}\right]\right|$.

Proof From Eq. (23), 


$$
\begin{aligned}
\sum_{j=0}^{n-1} & \sum_{i=0}^{N}\left(\left(l_{t}^{j+i \alpha} D_{t}^{j+i \alpha} w\right)(x, t)-\left(l_{t}^{j+(i+1) \alpha} D_{t}^{j+(i+1) \alpha} w\right)(x, t)\right) \\
= & \sum_{j=0}^{n-1} \sum_{i=0}^{N} \frac{\left(t-t_{0}\right)^{j+i \alpha}}{\Gamma(j+i \alpha+1)} D_{t}^{j+i \alpha} w\left(x, t_{0}\right) \\
& =\sum_{j=0}^{n-1} \sum_{i=0}^{N} w_{j+i \alpha}(x)\left(t-t_{0}\right)^{j+i \alpha} .
\end{aligned}
$$

That is,

$$
\begin{gathered}
w(x, t)-\sum_{j=0}^{n-1}\left[\left(t_{t}^{j+(N+1) \alpha} D_{t}^{j+(N+1) \alpha} w\right)(x, t)\right] \\
=\sum_{j=0}^{n-1} \sum_{i=0}^{N} W_{j+i \alpha}(x)\left(t-t_{0}\right)^{j+i \alpha} .
\end{gathered}
$$

Considering the second term of Eq. (27), we have

$$
\begin{aligned}
\sum_{j=0}^{n-1} & {\left[\left(l_{t}^{j+(N+1) \alpha} D_{t}^{j+(N+1) \alpha} w\right)(x, t)\right] } \\
= & \sum_{j=0}^{n-1}\left[\frac{1}{\Gamma((N+1) \alpha+j)} \int_{0}^{t} \frac{D^{j+(N+1) \alpha} w(x, \varepsilon)}{(t-\tau)^{1-(j+(N+1) \alpha)}} d \tau\right], \\
= & \sum_{j=0}^{n-1}\left[\frac{D^{j+(N+1) \alpha} w(x, \varepsilon)}{\Gamma((N+1) \alpha+j+1)} t^{(j+(N+1) \alpha)}\right] .
\end{aligned}
$$

(Mean value theorem for integral)

From Eqs. (27) and (28),

$$
\begin{aligned}
& w(x, t)-\sum_{j=0}^{n-1} \sum_{i=0}^{N} W_{j+i \alpha}(x)\left(t-t_{0}\right)^{j+i \alpha} \\
& =\sum_{j=0}^{n-1}\left[\frac{D^{j+(N+1) \alpha} W(x, \varepsilon)}{\Gamma((N+1) \alpha+j+1)} t^{(j+(N+1) \alpha)}\right]
\end{aligned}
$$

Now the error term is

$$
\begin{aligned}
\left\|E_{N}(x, t)\right\| & =\left\|w(x, t)-\sum_{j=0}^{n-1} \sum_{i=0}^{N} W_{j+i \alpha}(x)\left(t-t_{0}\right)^{j+i \alpha}\right\|, \\
& =\left\|\sum_{j=0}^{n-1}\left[\frac{D^{j+(N+1) \alpha} w(x, \varepsilon)}{\Gamma((N+1) \alpha+j+1)} t^{(j+(N+1) \alpha)}\right]\right\| \\
& \Rightarrow\left\|E_{N}(x, t)\right\| \\
& =\sup _{t \in[0, T]}\left|\sum_{j=0}^{n-1}\left[\frac{D^{j+(N+1) \alpha} w(x, \varepsilon)}{\Gamma((N+1) \alpha+j+1)} t^{(j+(N+1) \alpha)}\right]\right|,
\end{aligned}
$$

As $N \rightarrow \infty,\left\|E_{N}(x, t)\right\| \rightarrow 0$, thus $w(x, t)$ can be estimated as followsw $(x, t) \cong \sum_{j=0}^{n-1} \sum_{i=0}^{N} W_{j+i \alpha}(x)\left(t-t_{0}\right)^{j+i \alpha}$, with the error term in Eq. (25).

\section{Numerical examples}

Example 1 Consider Eqs. (12) and (13)

According to the RPSM, $w_{0}(x, t)=\max \left(0, e^{x}-1\right)$ and the infinite series solution of Eq. (12) can be written as

$w(x, t)=\max \left(0, e^{x}-1\right)+\sum_{k=1}^{\infty} b_{k}(x) \frac{t^{\alpha k}}{\Gamma(\alpha k+1)}$.

mth truncated series solution of $w(x, t)$ becomes

$w_{m}(x, t)=\max \left(0, e^{x}-1\right)+\sum_{k=1}^{m} b_{k}(x) \frac{t^{\alpha k}}{\Gamma(\alpha k+1)}, \quad m=1,2,3, \ldots$,

For $m=1$, 1st RPS solution for Eq. (11) may be written as

$w_{1}(x, t)=\max \left(0, e^{x}-1\right)+b_{1}(x) \frac{t^{\alpha}}{\Gamma(\alpha+1)}$.

To determine the value of $b_{1}(x)$, we substitute Eq. (31) in the $1 \mathrm{st}$ residual function of Eq. (18) $\operatorname{res}_{1}(x, t)=\frac{\partial^{\alpha} w_{1}(x, t)}{\partial t^{\alpha}}-\frac{\partial^{2} w_{1}(x, t)}{\partial x^{2}}-(k-1) \frac{\partial w_{1}(x, t)}{\partial x}+k w_{1}(x, t)$, this gives

$$
\begin{aligned}
\operatorname{res}_{1}(x, t)= & b_{1}(x)-e^{x}-b_{1}^{\prime \prime}(x) \frac{t^{\alpha}}{\Gamma(\alpha+1)} \\
& -(k-1)\left(e^{x}+b_{1}^{\prime}(x) \frac{t^{\alpha}}{\Gamma(\alpha+1)}\right) \\
& +k\left(\max \left(0, e^{x}-1\right)+b_{1}(x) \frac{t^{\alpha}}{\Gamma(\alpha+1)}\right) .
\end{aligned}
$$

Using (iii) of Eq. (19) for $i=0$ that is $\operatorname{res}(x, 0)=\operatorname{res}_{1}(x, 0)=0$, we get

$\operatorname{res}_{1}(x, 0)=b_{1}(x)-k e^{x}+k \max \left(e^{x}-1,0\right)=0$.

So $b_{1}(x)=k e^{x}-k \max \left(e^{x}-1,0\right)$.

For $m=2$, 2nd RPS solution for Eq. (11) can be written as

$$
\begin{aligned}
w_{2}(x, t)= & \max \left(e^{x}-1,0\right)+\left(k e^{x}-k \max \left(e^{x}-1,0\right)\right) \\
& \times \frac{t^{\alpha}}{\Gamma(\alpha+1)}+b_{2}(x) \frac{t^{2 \alpha}}{\Gamma(2 \alpha+1)} .
\end{aligned}
$$

To find the value of $b_{2}(x)$, Eq. (34) is substituted in the 2 nd residual function of Eq. (18) $\operatorname{res}_{2}(x, t)=\frac{\partial^{\alpha} W_{2}(x, t)}{\partial t^{\alpha}}-\frac{\partial^{2} w_{2}(x, t)}{\partial x^{2}}-(k-1) \frac{\partial w_{2}(x, t)}{\partial x}+k w_{2}(x, t)$. Then we have 


$$
\begin{aligned}
& \operatorname{res}_{2}(x, t)=k e^{x}-k \max \left(0, e^{x}-1\right)+b_{2}(x) \frac{t^{\alpha}}{\Gamma(\alpha+1)}-e^{x} \\
& \quad-b_{2}^{\prime \prime}(x) \frac{t^{2 \alpha}}{\Gamma(2 \alpha+1)}-(k-1)\left[e^{x}+b_{2}^{\prime}(x) \frac{t^{2 \alpha}}{\Gamma(2 \alpha+1)}\right] \\
& +k\left[\max \left(e^{x}-1,0\right)+\left(k e^{x}-k \max \left(e^{x}-1,0\right)\right) \frac{t^{\alpha}}{\Gamma(\alpha+1)}\right. \\
& \left.\quad+b_{2}(x) \frac{t^{2 \alpha}}{\Gamma(2 \alpha+1)}\right]
\end{aligned}
$$

Using (iii) of Eq. (19) for $i=1$ that is $D_{t}^{\alpha} \operatorname{res}(x, 0)=D_{t}^{\alpha} \operatorname{res}_{2}(x, 0)=0$, we get

$$
b_{2}(x)=k^{2} \max \left(0, e^{x}-1\right)-k^{2} e^{x} \text {. }
$$

For $m=3$, 3rd RPS solution for the Eq. (11) can be written as

$$
\begin{aligned}
w_{3}(x, t)= & \max \left(0, e^{x}-1\right)+\left(k e^{x}-k \max \left(0, e^{x}-1\right)\right) \\
& \times \frac{t^{\alpha}}{\Gamma(\alpha+1)}+\left(k^{2} \max \left(e^{x}-1,0\right)-k^{2} e^{x}\right) \\
& \times \frac{t^{2 \alpha}}{\Gamma(2 \alpha+1)}+b_{3}(x) \frac{t^{3 \alpha}}{\Gamma(3 \alpha+1)} .
\end{aligned}
$$

$$
\begin{aligned}
= & \max \left(e^{x}-1,0\right)\left[1-\frac{k t^{\alpha}}{\Gamma(1+\alpha)}+\frac{k^{2} t^{2 \alpha}}{\Gamma(1+2 \alpha)}-\frac{k^{3} t^{3 \alpha}}{\Gamma(1+3 \alpha)}+\cdots\right] \\
& +e^{x}\left[\frac{k t^{\alpha}}{\Gamma(1+\alpha)}-\frac{k^{2} t^{2 \alpha}}{\Gamma(1+2 \alpha)}+\frac{k^{3} t^{3 \alpha}}{\Gamma(1+3 \alpha)}-\cdots\right] \\
= & \max \left(e^{x}-1,0\right) E_{\alpha}\left(-k t^{\alpha}\right)+e^{x}\left(1-E_{\alpha}\left(-k t^{\alpha}\right)\right) .
\end{aligned}
$$

where $E_{\alpha}(t)=\sum_{n=0}^{\infty} \frac{t^{n}}{\Gamma(1+n \alpha)}$, is Mittag-Leffler function. Equation (42) is the analytical solution of Eq. (11) which is same as [10-13].

Case 1 Considering the vanilla call option [25] for $\alpha=1, \sigma=0.2, r=0.04, \tau=0.5$. years then $k=2$. The solution of Eq. (11) for this case is $w(x, t)=\max \left(0, e^{x}-1\right) e^{-2 t}+e^{x}\left(1-e^{-2 t}\right)$.

Case 2 For vanilla call option [25] with parameter $\sigma=0.2, r=0.01, \alpha=1, \tau=1$ year then $k=5$. In this example, we obtain the solution of Eq. (11) as $w(x, t)=\max \left(0, e^{x}-1\right) e^{-5 t}+e^{x}\left(1-e^{-5 t}\right)$.

Example 2 Let us consider the generalized BS equation [24]

Putting Eq. (37) in the $3 r d$ residual function of Eq. (18) we obtain $\operatorname{res}_{3}(x, t)=\frac{\partial^{\alpha} W_{3}(x, t)}{\partial t^{\alpha}}-\frac{\partial^{2} W_{3}(x, t)}{\partial x^{2}}-(k-1) \frac{\partial W_{3}(x, t)}{\partial x}+k w_{3}(x, t)$, we have

$$
\begin{aligned}
\operatorname{res}_{3}(x, t)= & k e^{x}-k \max \left(e^{x}-1,0\right)+\left(k^{2} \max \left(e^{x}-1,0\right)-k^{2} e^{x}\right) \frac{t^{\alpha}}{\Gamma(\alpha+1)} \\
& +b_{3}(x) \frac{t^{2 \alpha}}{\Gamma(2 \alpha+1)}-e^{x}-b_{3}^{\prime \prime}(x) \frac{t^{3 \alpha}}{\Gamma(3 \alpha+1)}-(k-1)\left[e^{x}+b_{3}^{\prime}(x) \frac{t^{3 \alpha}}{\Gamma(3 \alpha+1)}\right] \\
& +k\left[\begin{array}{l}
\max \left(0, e^{x}-1\right)+\left(k e^{x}-k \max \left(0, e^{x}-1\right)\right) \frac{t^{\alpha}}{\Gamma(\alpha+1)} \\
+\left(k^{2} \max \left(0, e^{x}-1\right)-k^{2} e^{x}\right) \frac{t^{2 \alpha}}{\Gamma(2 \alpha+1)}+b_{3}(x) \frac{t^{3 \alpha}}{\Gamma(3 \alpha+1)}
\end{array}\right] .
\end{aligned}
$$

Using Eq. (19) for $i=2$ that is $D_{t}^{2 \alpha} \operatorname{res}(x, 0)=D_{t}^{2 \alpha} \operatorname{res}_{3}(x, 0)=0$, it follows that

$b_{3}(x)=k^{3} e^{x}-k^{3} \max \left(e^{x}-1,0\right)$.

Continuing this way, one may find the values of $b_{4}(x)$, $b_{5}(x), \ldots .$. So the solution of Eq. (11) may be written as

$$
\begin{aligned}
w(x, t)= & \max \left(0, e^{x}-1\right)+\left(k e^{x}-k \max \left(0, e^{x}-1\right)\right) \frac{t^{\alpha}}{\Gamma(\alpha+1)} \\
& +\left(k^{2} \max \left(0, e^{x}-1\right)-k^{2} e^{x}\right) \frac{t^{2 \alpha}}{\Gamma(2 \alpha+1)} \\
& +\left(k^{3} e^{x}-k^{3} \max \left(0, e^{x}-1\right)\right) \frac{t^{3 \alpha}}{\Gamma(3 \alpha+1)}+\cdots
\end{aligned}
$$

$$
\begin{aligned}
& \frac{\partial^{\alpha} w}{\partial t^{\alpha}}+0.08(2+\sin x)^{2} x^{2} \frac{\partial^{2} w}{\partial x^{2}} \\
& \quad+0.06 x \frac{\partial w}{\partial x}-0.06 w=0, \quad 0<\alpha \leq 1,
\end{aligned}
$$

with IC

$$
w(x, 0)=\max \left(0, x-25 e^{-0.06}\right) .
$$

According to the RPSM, $w_{0}(x, t)=\max \left(0, x-25 e^{-0.06}\right)$, and the FPS solution of Eq. (43) can be written as

$$
w(x, t)=\max \left(x-25 e^{-0.06}, 0\right)+\sum_{k=1}^{\infty} b_{k}(x) \frac{t^{\alpha k}}{\Gamma(\alpha k+1)} .
$$


In consideration of the present method, mth truncated series solution of $w(x, t)$ becomes

$$
\begin{aligned}
w_{m}(x, t)= & \max \left(0, x-25 e^{-0.06}\right) \\
& +\sum_{k=1}^{m} b_{k}(x) \frac{t^{\alpha k}}{\Gamma(\alpha k+1)}, \quad m=1,2,3, \ldots,
\end{aligned}
$$

and mth residual function of Eq. (43) may be written as

$$
\begin{aligned}
& \operatorname{res}_{m}(x, t)=\frac{\partial^{\alpha} w_{m}(x, t)}{\partial t^{\alpha}}+0.08(2+\sin x)^{2} x^{2} \frac{\partial^{2} w_{m}(x, t)}{\partial x^{2}} \\
& +0.06 x \frac{\partial w_{m}(x, t)}{\partial x}-0.06 w_{m}(x, t), \quad m=1,2,3, \ldots,
\end{aligned}
$$

For $m=1$, 1st RPS solution for the Eq. (43) can be written as

$w_{1}(x, t)=\max \left(x-25 e^{-0.06}, 0\right)+b_{1}(x) \frac{t^{\alpha}}{\Gamma(\alpha+1)}$.

By substituting Eq. (48) into the 1st residual function of Eq. (47) as

$$
\begin{aligned}
\operatorname{res}_{1}(x, t)= & \frac{\partial^{\alpha} w_{1}(x, t)}{\partial t^{\alpha}}+0.08(2+\sin x)^{2} x^{2} \frac{\partial^{2} w_{1}(x, t)}{\partial x^{2}} \\
& +0.06 x \frac{\partial w_{1}(x, t)}{\partial x}-0.06 w_{1}(x, t),
\end{aligned}
$$

We get

$$
\begin{aligned}
\operatorname{res}_{1}(x, t)= & b_{1}(x)+0.08(2+\sin x)^{2} x^{2} b_{1}^{\prime \prime}(x) \frac{t^{\alpha}}{\Gamma(1+\alpha)} \\
& +0.06 x\left(1+b_{1}^{\prime}(x) \frac{t^{\alpha}}{\Gamma(1+\alpha)}\right) \\
& -0.06\left(\max \left(x-25 e^{-0.06}, 0\right)+b_{1}(x) \frac{t^{\alpha}}{\Gamma(\alpha+1)}\right) .
\end{aligned}
$$

Using (iii) of Eq. (19) for $i=0$ that is $\operatorname{res}(x, 0)=\operatorname{res}_{1}(x, 0)=0$, we get

$b_{1}(x)+0.06 x-0.06 \max \left(x-25 e^{-0.06}, 0\right)=0$.

$b_{1}(x)=0.06 \max \left(x-25 e^{-0.06}, 0\right)-0.06 x$.
For $m=2$, 2nd RPS solution for the Eq. (43) is written as

$$
\begin{aligned}
w_{2}(x, t)= & \max \left(0, x-25 e^{-0.06}\right) \\
& +\left(0.06 \max \left(0, x-25 e^{-0.06}\right)-0.06 x\right) \\
& \times \frac{t^{\alpha}}{\Gamma(\alpha+1)}+b_{2}(x) \frac{t^{2 \alpha}}{\Gamma(2 \alpha+1)},
\end{aligned}
$$

To find out the value of $b_{2}(x)$, substitute Eq. (52) into the 2 nd residual function of Eq. (47) as $\operatorname{res}_{2}(x, t)=\frac{\partial^{\alpha} w_{2}(x, t)}{\partial t^{\alpha}}+0.08(2+\sin x)^{2} x^{2} \frac{\partial^{2} w_{2}(x, t)}{\partial x^{2}}+0.06 x$ $\frac{\partial w_{2}(x, t)}{\partial x}-0.06 w_{2}(x, t)$, we get

$$
\begin{aligned}
\operatorname{res}_{2}(x, t)= & 0.06 \max \left(x-25 e^{-0.06}, 0\right)-0.06 x+b_{2}(x) \frac{t^{\alpha}}{\Gamma(1+\alpha)} \\
& +0.08(2+\sin x)^{2} x^{2}\left(b_{2}^{\prime \prime}(x) \frac{t^{2 \alpha}}{\Gamma(1+2 \alpha)}\right) \\
& +0.06 x\left(1+b_{2}^{\prime}(x) \frac{t^{2 \alpha}}{\Gamma(2 \alpha+1)}\right) \\
& -0.06\left(\begin{array}{l}
\max \left(x-25 e^{-0.06}, 0\right) \\
+\left(0.06 \max \left(x-25 e^{-0.06}, 0\right)-0.06 x\right) \\
\times \frac{t^{\alpha}}{\Gamma(\alpha+1)}+b_{2}(x) \frac{t^{2 \alpha}}{\Gamma(2 \alpha+1)}
\end{array}\right) .
\end{aligned}
$$

Using (iii) of Eq. (19) for $i=1$ that is $D_{t}^{\alpha} \operatorname{res}(x, 0)=$ $D_{t}^{\alpha} \operatorname{res}_{2}(x, 0)=0$, we get

$b_{2}(x)-0.06\left(0.06 \max \left(x-25 e^{-0.06}, 0\right)-0.06 x\right)=0$.

$$
\begin{aligned}
b_{2}(x) & =0.06\left(0.06 \max \left(0, x-25 e^{-0.06}\right)-0.06 x\right) \\
& =(0.06)^{2} \max \left(0, x-25 e^{-0.06}\right)-(0.06)^{2} x .
\end{aligned}
$$

For $m=3$, 3rd RPS solution for the Eq. (43) is reduced as

$$
\begin{aligned}
w_{3}(x, t)= & \max \left(x-25 e^{-0.06}, 0\right) \\
& +\left(0.06 \max \left(0, x-25 e^{-0.06}\right)-0.06 x\right) \\
& \frac{t^{\alpha}}{\Gamma(\alpha+1)}+\left((0.06)^{2} \max \left(0, x-25 e^{-0.06}\right)\right. \\
& \left.-(0.06)^{2} x\right) \frac{t^{2 \alpha}}{\Gamma(2 \alpha+1)}+b_{3}(x) \frac{t^{3 \alpha}}{\Gamma(3 \alpha+1)} .
\end{aligned}
$$

To determine the value of $b_{3}(x)$, putting Eq. (55) into the 3rd residual function of Eq. (47) as $\operatorname{res}_{3}(x, t)=\frac{\partial^{\alpha} w_{3}(x, t)}{\partial t^{\alpha}}+(2+\sin x)^{2} x^{2} 0.08 \frac{\partial^{2} w_{3}(x, t)}{\partial x^{2}}+0.06 x$ $\frac{\partial w_{3}(x, t)}{\partial x}-0.06 w_{3}(x, t)$, one may get

$$
\begin{aligned}
\operatorname{res}_{3}(x, t)= & 0.06 \max \left(0, x-25 e^{-0.06}\right)-0.06 x+\left((0.06)^{2} \max \left(0, x-25 e^{-0.06}\right)-(0.06)^{2} x\right) \frac{t^{\alpha}}{\Gamma(1+\alpha)} \\
& +b_{3}(x) \frac{t^{2 \alpha}}{\Gamma(1+2 \alpha)}+(2+\sin x)^{2} 0.08 x^{2}\left(b_{3}^{\prime \prime}(x) \frac{t^{3 \alpha}}{\Gamma(1+3 \alpha)}\right)+0.06 x\left(1+b_{3}^{\prime}(x) \frac{t^{3 \alpha}}{\Gamma(3 \alpha+1)}\right) \\
& -0.06\left(\begin{array}{l}
\max \left(0, x-25 e^{-0.06}\right)+\left(0.06 \max \left(0, x-25 e^{-0.06}\right)-0.06 x\right) \frac{t^{\alpha}}{\Gamma(\alpha+1)} \\
+\left((0.06)^{2} \max \left(0, x-25 e^{-0.06}\right)-(0.06)^{2} x\right) \frac{t^{2 \alpha}}{\Gamma(2 \alpha+1)}+b_{3}(x) \frac{t^{3 \alpha}}{\Gamma(3 \alpha+1)}
\end{array}\right) .
\end{aligned}
$$




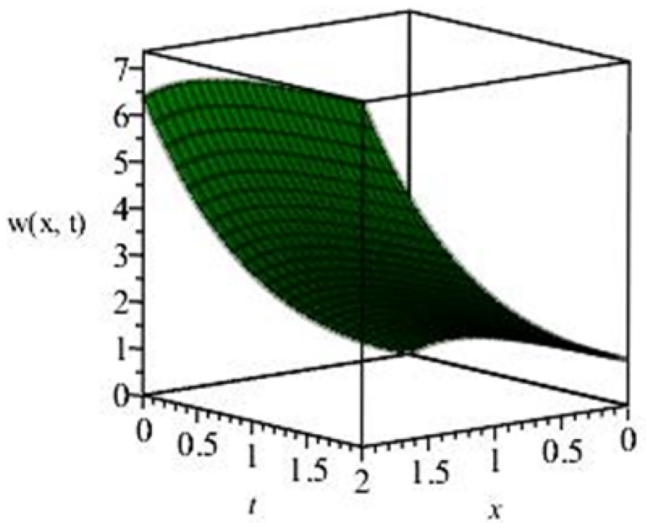

(a)

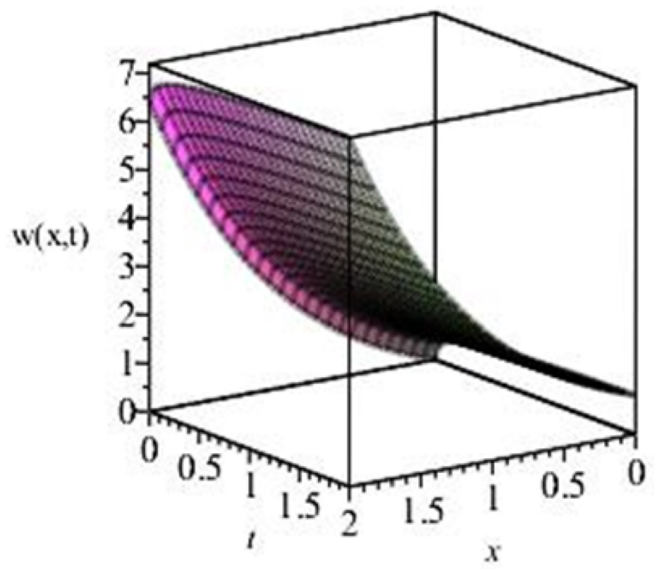

(c)

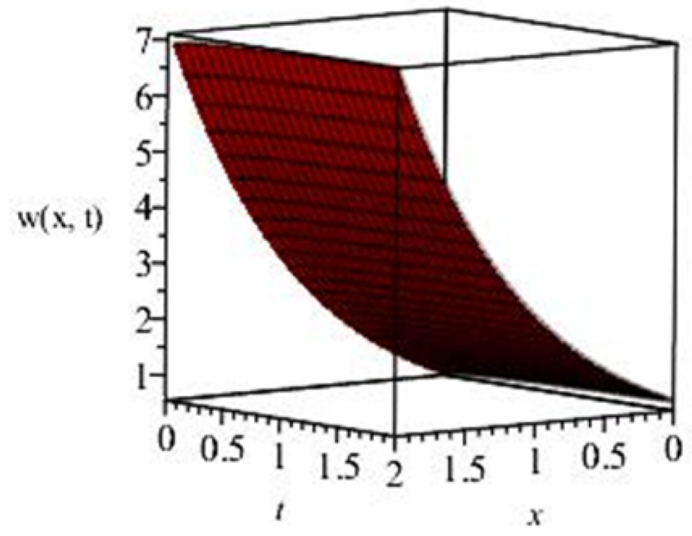

(b)

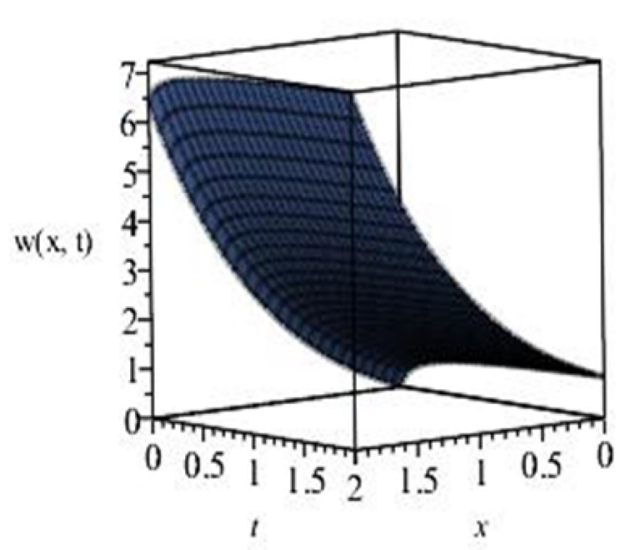

(d)

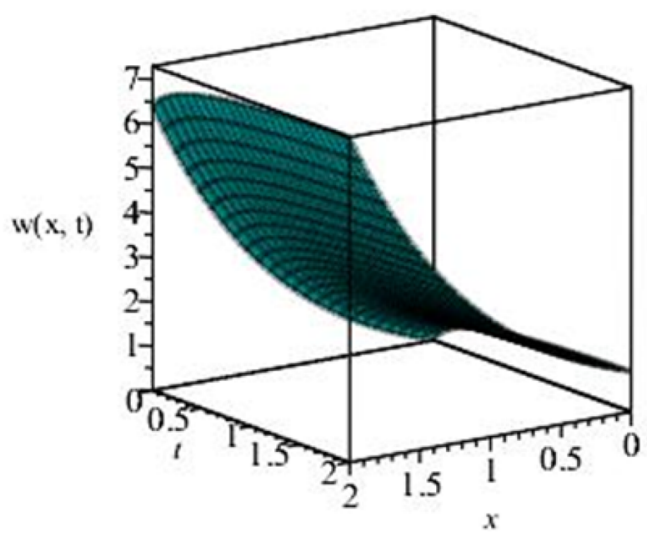

(e)

Fig. 1 The plot of Eq. (42) represents the surface $w(x, t)$ at $\mathbf{a} \alpha=1, \mathbf{b} \alpha=0.2, \mathbf{c} \alpha=0.5, \mathbf{d} \alpha=0.6$ and e $\alpha=0.8$

By Eq. (19) for $i=2$ that is $D_{t}^{2 \alpha} \operatorname{res}(x, 0)=D_{t}^{2 \alpha} \operatorname{res}_{3}(x, 0)=0$, it follows that

$$
\begin{aligned}
& b_{3}(x)-0.06\left((0.06)^{2} \max \left(0, x-25 e^{-0.06}\right)-(0.06)^{2} x\right)=0 \\
& b_{3}(x)=(0.06)^{3} \max \left(0, x-25 e^{-0.06}\right)-(0.06)^{3} x
\end{aligned}
$$




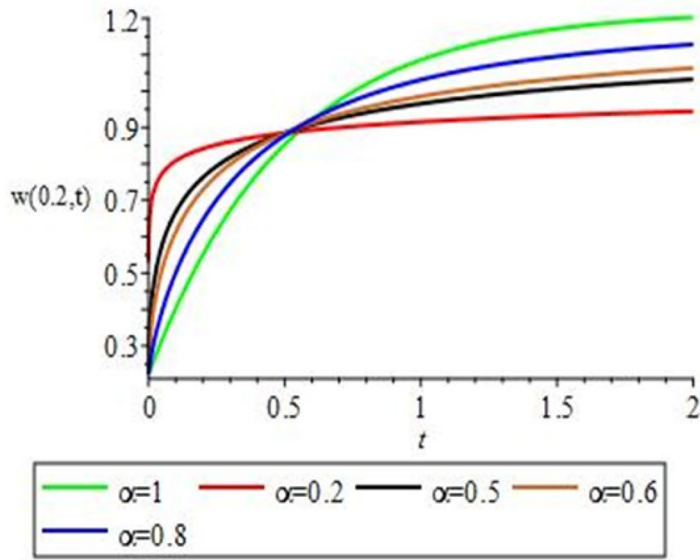

(a)

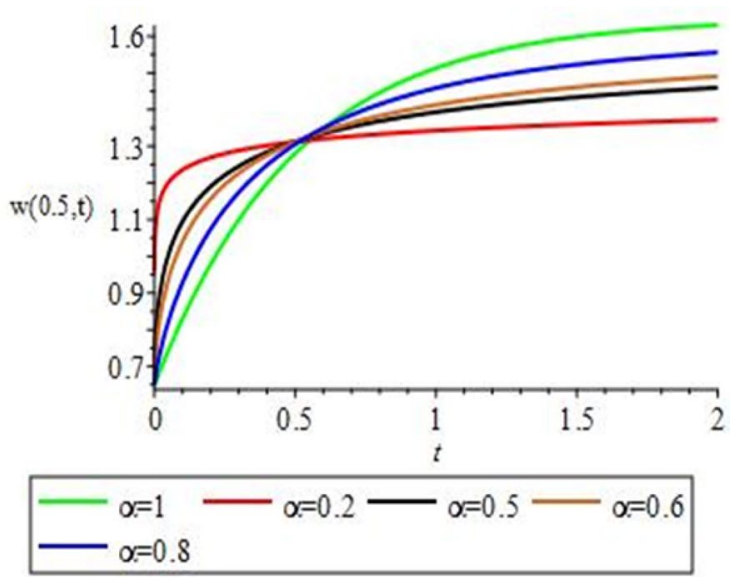

(b)

Fig. 2 The solution plots of Eq. (42) for different values of $\alpha$ at $\mathbf{a} x=0.2, \mathbf{b} x=0.5$

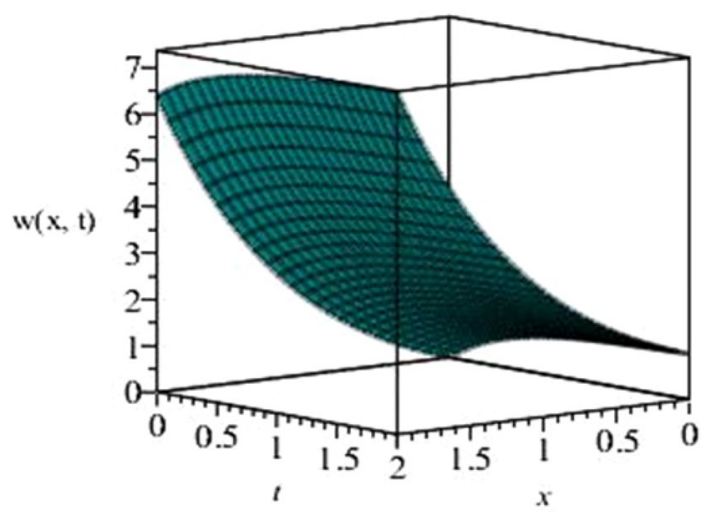

Fig. 3 The plot represents the surface $w(x, t)$ at $\alpha=1$ and $k=2$

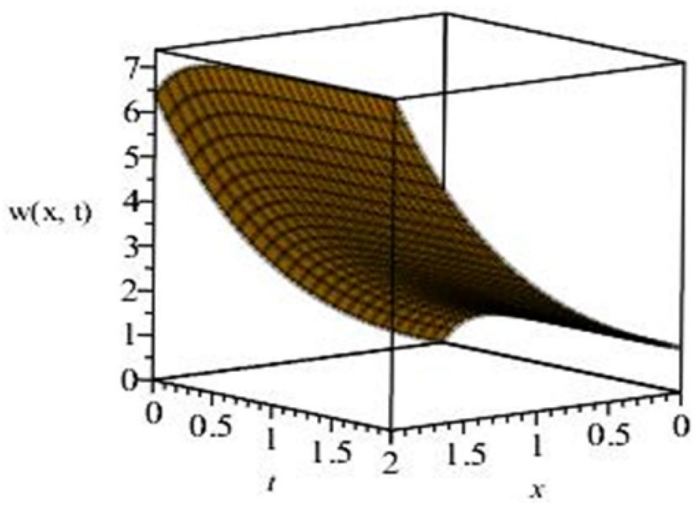

Fig. 4 The plot represents the surface $w(x, t)$ at $\alpha=1$ and $k=5$

Continuing as above, one may obtain the values of $b_{4}(x)$, $b_{5}(x), \ldots \ldots$, So the solution of Eq. (43) may be written as

$$
\begin{aligned}
w(x, t)= & \max \left(0, x-25 e^{-0.06}\right)+\left(0.06 \max \left(0, x-25 e^{-0.06}\right)-0.06 x\right) \frac{t^{\alpha}}{\Gamma(\alpha+1)} \\
& +\left((0.06)^{2} \max \left(0, x-25 e^{-0.06}\right)-(0.06)^{2} x\right) \frac{t^{2 \alpha}}{\Gamma(2 \alpha+1)} \\
& +\left((0.06)^{3} \max \left(0, x-25 e^{-0.06}\right)-\right) \frac{t^{3 \alpha}}{\Gamma(3 \alpha+1)}+\cdots, \\
= & \max \left(0, x-25 e^{-0.06}\right)\left[1+\frac{0.06 t^{\alpha}}{\Gamma(1+\alpha)}+\frac{(0.06)^{2} t^{2 \alpha}}{\Gamma(1+2 \alpha)}+\frac{(0.06)^{3} t^{3 \alpha}}{\Gamma(1+3 \alpha)}+\cdots\right] \\
& +x\left[-\frac{0.06 t^{\alpha}}{\Gamma(1+\alpha)}-\frac{(0.06)^{2} t^{2 \alpha}}{\Gamma(1+2 \alpha)}-\frac{(0.06)^{3} t^{3 \alpha}}{\Gamma(1+3 \alpha)}-\cdots\right] \\
= & \max \left(0, x-25 e^{-0.06}\right) E_{\alpha}\left(0.06 t^{\alpha}\right)+x\left(1-E_{\alpha}\left(0.06 t^{\alpha}\right)\right) .
\end{aligned}
$$




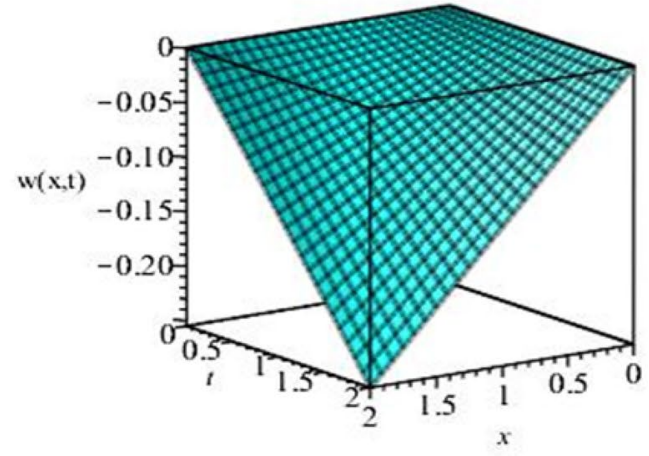

(a)

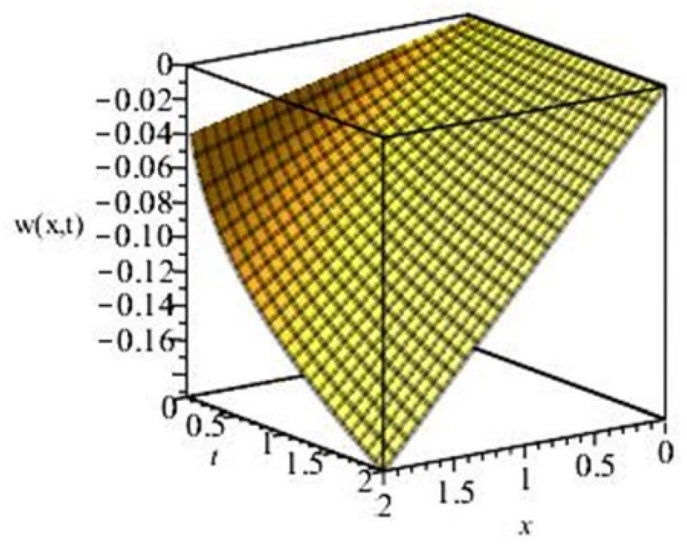

(c)

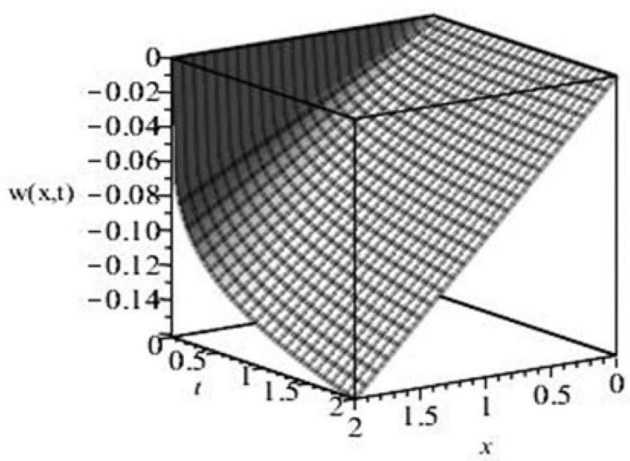

(b)

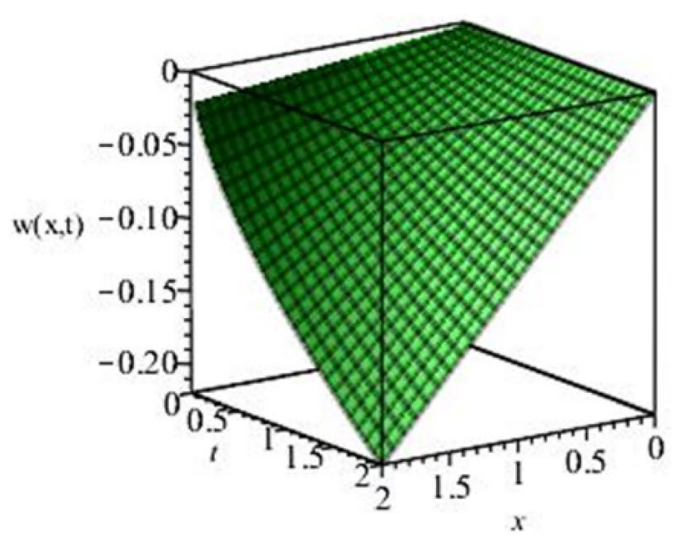

(d)

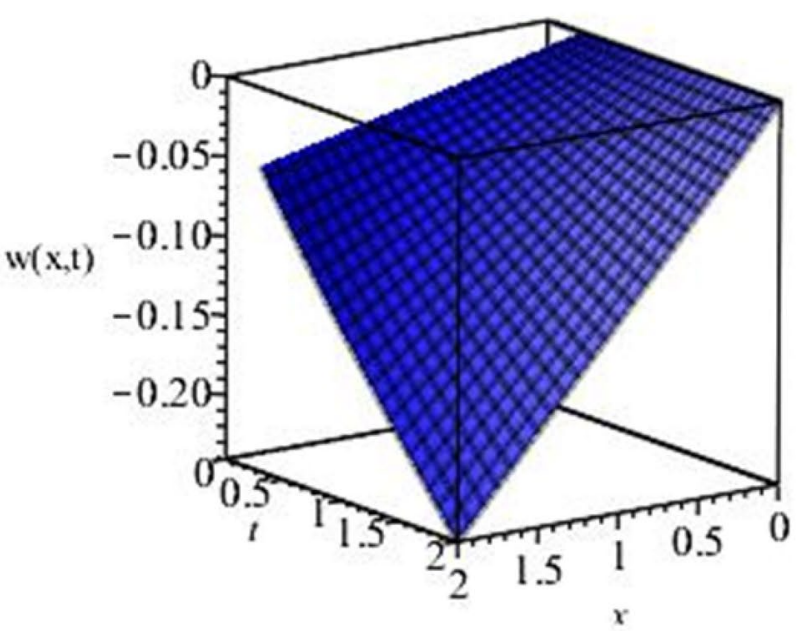

(e)

Fig. 5 The plot of Eq. (58) represents the surface $w(x, t)$ at $\mathbf{a} \alpha=1, \mathbf{b} \alpha=0.2, \mathbf{c} \alpha=0.4, \mathbf{d} \alpha=0.6$ and e $\alpha=0.8$

Equation (58) is the exact solution of Eq. (43) which is the same as given in [11]. For $\alpha=1$, we have $w(x, t)=\max \left(x-25 e^{-0.06}, 0\right) e^{0.06 t}+x\left(1-e^{0.06 t}\right)$. This is an analytical solution of fractional BS Eq. (43).

\section{Conclusion}

In this study, a new iterative technique namely RPSM is effectively applied for finding the exact solution of FBSE with high accuracy. The convergence analysis is also 


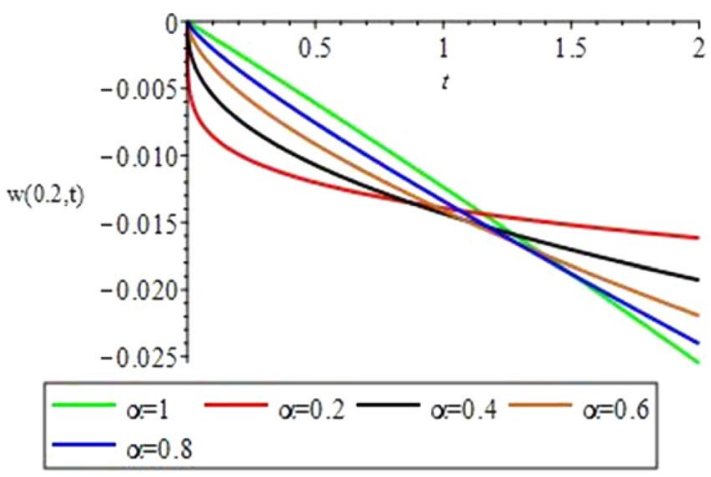

(a)

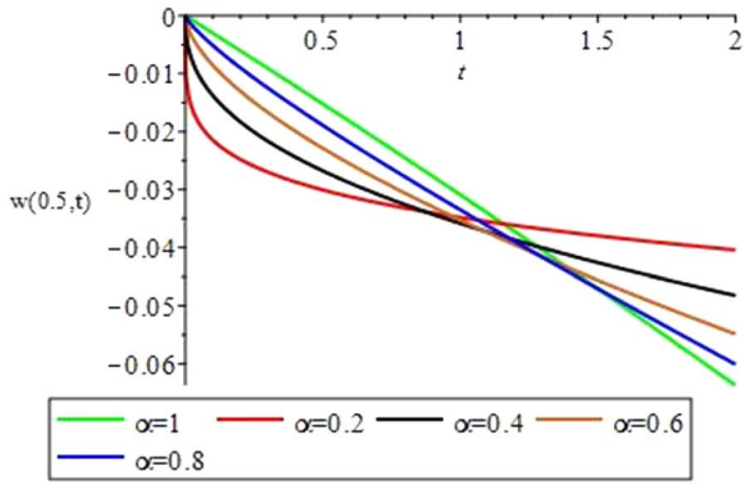

(b)

Fig. 6 The solution plots of Eq. (58) for different values of $\alpha$ at $\mathbf{a} x=0.2$ and $\mathbf{b} x=0.5$

described to validate the efficacy and powerfulness of the present technique. Solution plots of Eqs. (42) and (58) have been illustrated in Figs. 1, 2, 3, 4, 5 and 6 for different values of $\alpha$. It is clear from the figures that the European option prices increase with the decrease of $\alpha$. Moreover at $\alpha=0.2$ it is seen that option is overpriced. The solutions in special cases achieved from the proposed method are in good agreement with the other methods described in [10-13] which shows that the method is effective, convenient and gives closed form solution in the series form.

Acknowledgements The first author would like to thank Department of Science and Technology, Govt. of India for giving INSPIRE fellowship (IF170207) to carry out the present work.

\section{Compliance with ethical standards}

Conflict of interest All authors state that they have no conflict of interest.

\section{References}

1. Black F, Scholes M (1973) The pricing of options and corporate liabilities. J Polit Econ 81:354-637

2. Manale JM, Mahomed FM (2000) A simple formula for valuing American and European call and put options. In: Banasiak J (ed) Proceeding of the Hanno Rund workshop on the differential equations. University of Natal, Durban, pp 210-220

3. Hilfer R (ed) (2000) Applications of fractional calculus in physics. World Scientific Publishing Company, Singapore, pp 87-130

4. Podlubny I (1999) Fractional differential equations. Academic Press, New York

5. Caputo M (1969) Elasticita e dissipazione. Zani-Chelli, Bologna

6. Miller KS, Ross B (1993) An introduction to the fractional calculus and fractional differential equations. Wiley, New York

7. Kilbas AA, Srivastava HM, Trujillo JJ (2006) Theory and applications of fractional differential equations. Elsevier Science Publishers, Amsterdam

8. Heydari MH, Hooshmandasl MR, Ghaini FMM, Cattani C (2016) Wavelets method for solving fractional optimal control problems. Appl Math Comput 286:139-154

\section{SN Applied Sciences}

9. Heydari MH, Hooshmandasl MR, Shakiba A, Cattani C (2016) An efficient computational method based on the hat functions for solving fractional optimal control problems. Tbilisi Math J 9:143-157

10. Kumar S, Yildirim A, Khan Y, Jafari H, Sayevand K, Wei L (2012) Analytical solution of fractional Black-Scholes European option pricing equation by using Laplace transform. J Fract Calc Appl 2:1-9

11. Kumar S, Kumar D, Singh J (2014) Numerical computation of fractional Black-Scholes equation arising in financial market. Egypt J Basic Appl Sci 1:177-183

12. Elbeleze AA, Kilıcman A, Taib BM (2013) Homotopy perturbation method for fractional Black-Scholes European option pricing equations using Sumudu transform. In: Mathematical problems in engineering, $\mathrm{p} 7$

13. Edeki SO, Ugbebor OO, Owoloko EA (2015) Analytical solutions of the Black-Scholes pricing model for european option valuation via a projected differential transformation method. Entropy 17:7510-7521

14. Yavuz M, Özdemir N (2018) A different approach to the European option pricing model with new fractional operator. Math Model Nat Phenom 13:1-12

15. Özdemir N, Yavuz M (2017) Numerical solution of fractional Black-Scholes equation by using the multivariate Padé approximation. Acta Phys Pol A 132:1050-1053

16. Yavuz M, Özdemir N (2018) A quantitative approach to fractional option pricing problems with decomposition series. Konuralp J Math 6:102-109

17. Yavuz M, Özdemir N (2018) European Vanilla option pricing model of fractional order without Singular Kernel. Fractal Fract 2(1):1-11

18. Abu Arqub O (2013) Series solution of fuzzy differential equations under strongly generalized differentiability. J Adv Res Appl Math 5:31-52

19. Abu Arqub O, El-Ajou A, Bataineh A, Hashim I (2013) A representation of the exact solution of generalized Lane Emden equations using a new analytical method. In: Abstract and applied analysis, $\mathrm{P} 10$

20. El-Ajou A, Abu Arqub A, Momani S (2015) Approximate analytical solution of the nonlinear fractional KdV-Burgers equation: $a$ new iterative algorithm. J Comput Phys 293:81-95

21. Alquran $M$ (2014) Analytical solutions of fractional foam drainage equation by residual power series method. Math Sci 8(4):153-160 
22. Hira T, Ghazala A (2017) Residual power series method for solving time-space-fractional Benney-Lin equation arising in falling film problems. J Appl Math Comput 55:683-708

23. Gulkac V (2010) The homotopy perturbation method for the Black-Scholes equation. J Stat Comput Simul 80:1349-1354
24. Cen Z, Le A (2011) A robust and accurate finite difference method for a generalized Black-Scholes equation. J Comput Appl Math 235:3728-3733

25. Company R, Navarro E, Pintos JR, Ponsoda E (2008) Numerical solution of linear and nonlinear Black-Scholes option pricing. Comput Math Appl 56:813-821 